\title{
Enteral tube feeding for head and neck cancer (HNC) patients treated in Oxford by surgery (S), radiotherapy (RT) or chemoradiotherapy (CRT): development of clinical guidelines
}

\author{
A. Howard and R. Shah \\ Department of Therapies and Dietetics, Oxford Cancer Centre, Churchill Hospital, Old Road, Headington, \\ Oxford, OX3 7LJ, UK
}

It is now widely accepted that patients who undergo wide-field RT (treatment to both sides) of the oropharynx or nasopharynx require a prophylactic gastrostomy tube due to the side-effects of treatment. Patients who undergo unilateral RT (treatment to one side) of the oropharynx experience side-effects to a lesser degree and therefore generally do not require a feeding tube. Chemotherapy is now offered to eligible patients' undergoing radical $\mathrm{RT}^{(1)}$ which exacerbates the side-effects of $\mathrm{RT}$, such as oral mucositis and xerostomia. It is unknown whether patients undergoing unilateral CRT are able to maintain oral nutrition or whether they should also be considered for insertion of a feeding tube prior to commencement of treatment.

National Institute of Clinical Excellence (NICE) guidelines state that gastrostomy feeding should be considered in people likely to need long term (4 weeks or more) enteral tube feeding ${ }^{(2)}$; and that there should be specific guidelines on the use, placement and management of gastrostomy tubes in HNC patients ${ }^{(1)}$. However there are no clinical guidelines on feeding tube placement according to RT or CRT treatment fields. The aim of this audit was to establish length of tube feeding following S, RT or CRT according to treatment field, to provide evidence-based guidelines on which patients should be considered for prophylactic gastrostomy tube insertion prior to RT in accordance with NICE guidance.

Data on diagnosis, tumour staging, treatment details, oral and enteral nutrition for $263 \mathrm{HNC}$ patients undergoing RT or CRT as part of their treatment was collected between 1 April 2006 and 6 January 2011 and entered onto an analytical database $\left(\mathrm{Chiron}^{\mathrm{TM}}\right)$. The data was stringently verified and extensively analysed to determine which patients should be considered for a prophylactic feeding tube.

4 out of 19 patients required gastrostomy feeding during unilateral CRT. The average feed duration was 210 days (range $70-300$ days). 3 patients resumed oral diet and had their feeding tubes removed and 1 patient continued to feed due to tumour recurrence. 7 laryngectomy patients had CRT and none required tube feeding. This could relate to the fact that laryngectomees are not at risk of aspiration.

\begin{tabular}{lc}
\hline Clinical guideline selection criteria for prophylactic gastrostomy tube insertion in RT and CRT & Bilateral field \\
\hline Unilateral field & All patients except laryngectomees \\
\hline Large oropharyngeal tumours that cross the midline & \\
Slow swallow rehabilitation post surgery & \\
Body mass index (BMI) $<18.5 \mathrm{~kg} \mathrm{~m}^{-2}$ & \\
Social issues affecting oral nutrition (e.g. isolation, alcohol dependence, depression) & \\
\hline
\end{tabular}

Patients undergoing unilateral CRT do not routinely require tube feeding. However, we have identified patients who are likely to struggle with eating and drinking during treatment and who should be considered for a prophylactic feeding tube. Each patient is considered individually using the above selection criteria and a decision regarding appropriate tube placement is made by the HNC multidisciplinary team. We have successfully implemented these guidelines in Oxford.

1. National Institute for Clinical Excellence (2004) Nutrition support in adults: oral nutrition support, enteral tube feeding and parenteral nutrition. http:// www.guidance.nice.org.uk/CG32.

2. National Institute for Clinical Excellence (2006) Guidance on cancer services: improving outcomes in head and neck cancers-the manual. http:// www.guidance.nice.org.uk/CSGHN. 